\title{
Detection of fast flying nanoparticles by light scattering over a large volume
}

\author{
F. Pettazzi*, S. Bäumer, J. van der Donck, A. Deutz \\ TNO, The Netherlands Organization for Applied Scientific Research, Stieltjesweg 1, 2328 CK Delft, \\ The Netherlands
}

\begin{abstract}
Detection of nanoparticles is of paramount importance for contamination control in ultra-clean systems. Light scattering is a well-known detection method which is applied in many different scientific and technology domains including atmospheric physics, environmental control, and biology. It allows contactless and remote detection of sub-micron size particles. However, methods for detecting a single fast moving particle smaller than $100 \mathrm{~nm}$ are lacking.

In the present work we report a preliminary design study of an inline large area detector for nanoparticles larger than 50 $\mathrm{nm}$ which move with velocities up to $100 \mathrm{~m} / \mathrm{s}$. The detector design is based on light scattering using commercially available components.

The presented design takes into account all challenges connected to the inline implementation of the scattering technique in the system: the need for the detector to have a large field of view to cover a volume with a footprint commensurate to an area of $100 \mathrm{~mm} \times 100 \mathrm{~mm}$, the necessity to sense nanoparticles transported at high velocity, and the requirement of large capture rate with a false detection as low as one false positive per week. The impact of all these stringent requirements on the expected sensitivity and performances of the device is analyzed by mean of a dedicated performance model.
\end{abstract}

Keywords: Light scattering, nanoparticles, contamination, inline detection, false positive

\section{INTRODUCTION}

Airborne contamination in the form of particulate is nowadays a matter of concern for many technology and research fields, ranging from thin film deposition to photolithography [1]. In all these applications, the presence of contaminating particles flying in close vicinity of sensitive surfaces can impair the quality of the process and introduce defects in the product to be developed. Typical examples of technologies necessitating extreme measures to reduce particle contamination are thin film deposition and photolithography, two processes that require high throughput in order to minimize the cost of the final product.

In both cases, the current system performances and required yield imply that even single particles at the nanoscale can introduce "killer defects" in the process when impinging on sensitive surfaces.

To minimize the occurrence of particle contamination, many of these systems are operated in a close environment in near vacuum condition, or under the presence of a gas purge. While continuous effort is put to prevent particle deposition, it is still considered desirable to develop a reliable method to detect nanoparticles in the vicinity of critical surfaces. This method may provide an efficient mitigation technique and reduce potential machine dwell time, by generating an early warning in case a contaminating particle is detected during operation.

Several techniques are available to allow detection of particles at the nanoscale, ranging from the introduction of charged devices in the vicinity of the area of interest [2,3] to the use of magnetic fields to deflect charged particles [4]. Many of these methods, however, produce perturbation in the volume under investigation, or even require the presence of specific hardware having either high electric and/or magnetic fields in the close vicinity of it. These features are particularly detrimental and unpractical, as in many cases the area to be sensed needs to be free from obstructions to achieve an optimized process. Conversely to all the aforementioned techniques, optical scattering [5-6], which is based on the interaction between the particle and a laser beam, provides an inherently contactless detection, because the detection instrumentation can be placed far away from the region where the light interacts with the particle.

Due to this specific property, the detection of particles using optical scattering has been selected for the present study, which reports the preliminary design of an in-line optical instrument suitable for operation in ultra-clean closed systems.

*federico.pettazzi@tno.nl; phone 0888862624; tno.n1/optics

Modeling Aspects in Optical Metrology V, edited by Bernd Bodermann, Karsten Frenner, Richard M. Silver, Proc. of SPIE Vol. 9526, 952600 · C 2015 SPIE · CCC code: 0277-786X/15/\$18 · doi: 10.1117/12.2186079 
The remainder of the paper is organized as follows: section 2 discusses the main requirements considered in the present study. In section 3 the preliminary design of the system is described, and the expected performances are reported. Finally, section 4 summarizes the obtained results.

\section{DRIVING REQUIREMENTS FOR PARTICLE DETECTOR}

The primary goal of the present investigation is to design an instrument capable of detecting stainless steel nanoparticles of dimensions of few tens of nanometers for contamination control in an ultra-clean system. The required size stems from the fact that few tens of nanometers particles impinging on sensitive surfaces can sensibly alter process fabrication in photolithographic processes and in thin film deposition, two technology fields of interest for the current instrument. At the same time, stainless steel (or aluminum) particles are assumed, because it is expected that within the systems of interest the main source of contamination is generated by the vacuum system itself.

In addition, all technologies of interest typically take place in closed vessels operating in vacuum conditions or in presence of a flow of ultra-pure gas. In case of operation under purging flow conditions, which is not uncommon in many of such systems, additional challenges arise: contaminating particles in a gas flow can in fact acquire a kinetic momentum in a specific direction, resulting in a lower interaction time with the laser beam used for the scattering measurement, and ultimately imposing additional timing constraints on the design of the setup.

Finally, target applications for an in-line particle detector have typically stringent requirements for what concern the actual production yield. As a result, an in-line instrument operating in such systems should be able to detect contamination events in a macroscopic footprint area with high capture rate and minimal false rate detection. For one of our applications, an economically feasible process is only possible when one undetected particle is present in at least 50100 days [7]. This is technically not feasible. However, when the particle can be detected, the economic impact can be reduced and 1 detected particle per day is permissible. This allows a false positive rate of 1 per week.

The requirements considered for the preliminary design of the particle detector are listed in Table 2.1. The values reported are derived from a specific user case. However the challenges connected with the fulfillment of many of the listed specifications are common to many different technologies, and therefore the results obtained in the remainder of the paper have a more general value.

Table 2.1 List of applicable requirements for the study of in-line particle detector

\begin{tabular}{|c|c|c|c|}
\hline Requirement & Value & Units & Note \\
\hline $\begin{array}{c}\text { Minimum detectable } \\
\text { particle size }\end{array}$ & 50 & $\mathrm{~nm}$ & $\begin{array}{c}\text { Stainless } \\
\text { steel/Aluminum }\end{array}$ \\
\hline Maximum speed of particle & 100 & $\mathrm{~m} / \mathrm{s}$ & $\mathrm{mm}^{2}$ \\
\hline Coverage area & $100 \times 100$ & $\mathrm{~Pa}$ & $\begin{array}{c}\text { Or lower in case of } \\
\text { vacuum systems }\end{array}$ \\
\hline Pressure levels & $1-10$ & & \\
\hline False positive rate & 1 per week & & \\
\hline
\end{tabular}

\section{DESIGN OF THE IN LINE PARTICLE DETECTOR}

Light scattering is a physical process that takes place when electromagnetic radiation interacts with a discontinuity, like a particle or a surface. As the electromagnetic field impinges on the particle, it induces the formation of an electromagnetic dipole, which by itself acts as a source of radiation, whose properties may differ from the ones of the initial field. Specifically, scattered radiation can display different direction and wavelength with respect of the incoming light. A typical geometrical configuration for the scattering event is reported in Figure 1: a light beam, in many cases emitted from a laser source, is shaped to fill an observation volume in close vicinity of a sensitive surface. When a particle 
crosses such volume, electromagnetic radiation is scattered at different angles, and may be collected by a suitable detector, placed at an observation angle with respect of the incoming light.

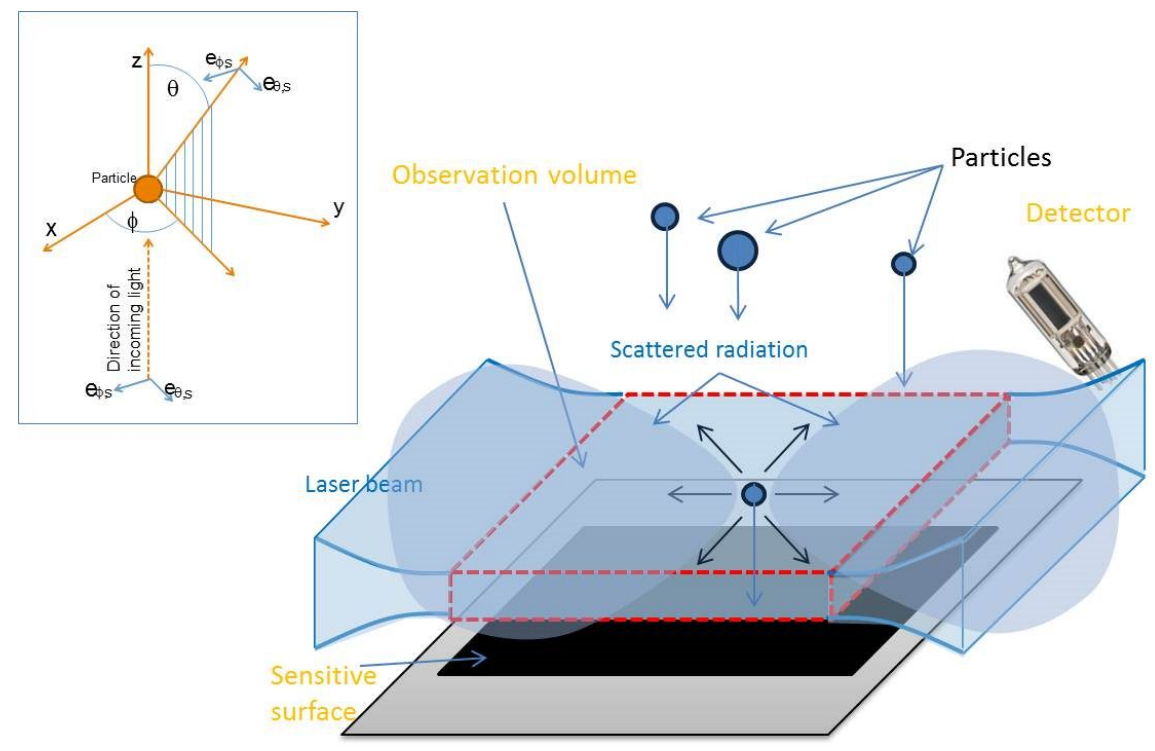

Figure 1 Principle setup for in-line particle detector using scattering. In the insert, the coordinate geometry for the Mie scattering is shown

In the specific case under investigation, particles as small as $50 \mathrm{~nm}$ can fly across the observation volume at a maximum velocity of $100 \mathrm{~m} / \mathrm{s}$. In those limit conditions, the expected scattering power will be extremely low, due to the combined detrimental effect of the small particle size and of the reduced interaction time, dictated by the high speed of the particle.

\section{Derivation of design guidelines for particle detector}

Design guidelines for the in line particle detector based on light scattering can be derived by the theory of Mie [5], which describes the interaction between light and spherical particles of variable size and composition. Such theory is therefore particularly suitable to analyze contamination phenomena, where a broad range of particle sizes is expected to be present.

The scattering of radiation from a spherical particle is related to the particle size and composition, to the wavelength and power density of the incident light, and to the geometric configuration of the problem, according to the relation

$$
P_{s c a}=\frac{P_{i} \lambda^{2} d A}{\pi^{3} D^{2} r^{2}}|S \cos \phi|^{2}
$$

being $\mathrm{Pi}, \lambda$ and $\mathrm{D}$ the incident power, the wavelength and the diameter of the beam, $\mathrm{dA}$ the area of the detector, $\mathrm{r}$ the distance between the particle and the detector, and $\mathrm{S}$ scattering coefficient, which is function of the particle size, complex refractive index and of the wavelength of the incoming light, and is determined numerically following the theory reported in [5]. While not apparent from Equation (1), in the limit of particles of size much smaller than the wavelength, the scattered power is inversely proportional to the fourth power of the wavelength, and proportional to the sixth power of the particle dimension - the Rayleigh scattering regime [5].

From Equation (1) it is possible to evaluate the driving parameters that can be tuned to increase the scattered power, and hence to enhance the efficiency of the in-line particle detector.

The first choice for increasing the scattering return is to impinge on the particle by using an high power laser source, which can be collimated to achieve a small spot diameter with high irradiance. However, a limit in the smaller spot size 
usable exists, due to the necessity of probing a relatively large volume on top of the sensitive surface, as shown in Table 2.1 .

Furthermore, operation in the UV or DUV spectral range is preferred, since it allows further increase of the scattering signal, due to the strong dependence of the scattering coefficient from the inverse of the wavelength. High peak power sources in the DUV or UV spectral band are commercially available by using pulsed solid state or fiber laser sources equipped with frequency conversion units [8] with power levels up to 20-30 W and repetition rates in the order of 100 $\mathrm{kHz}$ or even $1 \mathrm{MHz}$. Those sources offer high flexibility for implementation in different research and industrial environments, and are therefore preferred over the commercial Excimer lasers.

An additional positive effect on the scattering signal can be obtained by increasing the solid angle for the observation of the scattering radiation, either by reducing the distance of the detector from the volume of interest or by increasing the detector area. The latter solution can be implemented by using Micro Channel Plate (MCP) photomultipliers [9], which provide a very fast time response with high sensitivity and large active area.

For what concern the signal acquisition and processing, the implementation of averaging schemes could bring a benefit to the detection efficiency by increasing the signal to noise ratio. In this respect, digital boxcar integrators are particularly suitable to be used with low duty cycle signals like those generated by pulsed lasers, effectively providing a way of performing integration and average of the pulses scattered by the particle [10].

In the following table, the main guidelines applied for the design of the detector are reported, together with the selected approach for increasing the sensitivity of the instrument.

Table 3.1 Summary of the guidelines applied for the design of the in-line particle detector

\begin{tabular}{|l|l|l|l|}
\hline Functional unit & \multicolumn{1}{|c|}{$\begin{array}{c}\text { Design guideline } \\
\text { description }\end{array}$} & $\begin{array}{c}\text { Possible design } \\
\text { guideline } \\
\text { implementation }\end{array}$ & \multicolumn{1}{|c|}{ Selection and explanation } \\
\hline Laser source & $\begin{array}{l}\text { Increase power density } \\
\text { on particle }\end{array}$ & $\mathrm{Pi}, \downarrow \mathrm{D}^{2}$ & $\begin{array}{l}\uparrow \mathrm{Pi} \text { as } \mathrm{D} \text { is limited by the need of } \\
\text { ensuring coverage of a large } \\
\text { observation volume. }\end{array}$ \\
\cline { 2 - 4 } & $\begin{array}{l}\text { Decrease wavelength of } \\
\text { pump laser }\end{array}$ & $\begin{array}{l}\text { UV lasers are available in pulsed } \\
\text { operation mode }\end{array}$ \\
\hline Detector & $\begin{array}{l}\text { Increase solid angle } \\
\text { observation }\end{array}$ & $\begin{array}{l}\uparrow \mathrm{dA}, \downarrow \mathrm{r}^{2} \\
\text { the system } \mathrm{r} \text { is limited by the geometry of }\end{array}$ \\
\hline $\begin{array}{l}\text { Data acquisition } \\
\text { and processing }\end{array}$ & Averaging preferred & Boxcar integrator & Digital boxcar integrator \\
\hline
\end{tabular}

\section{Design description and performances of the in-line particle detector}

A descriptive view of the design of the in-line particle detector is shown in Figure 2.

The laser source considered is a fiber solid state laser frequency tripled, which can emit $30 \mathrm{uJ}$ pulses at a repetition rate of $1 \mathrm{MHz}$ and at a wavelength of $355 \mathrm{~nm}$. The laser is shaped to form a beam of $5 \mathrm{~mm}$ diameter and is reflected back and forth between two series of high reflectance dielectric mirrors in order to completely cover the observation volume. The chosen settings allow the full coverage of a footprint of 100 by $100 \mathrm{~mm}$ with 20 passages, corresponding to 40 reflections using mirrors in a zig-zag configuration. Assuming a nominal reflectance of about $99.7 \%$ per mirror, the total transmission losses experienced by the laser amount to $88.7 \%$.

With the presented design, a nanoparticle travelling at the maximum velocity of $100 \mathrm{~m} / \mathrm{s}$ crosses the $5 \mathrm{~mm}$ thick observation volume in a time of $50 \mu \mathrm{s}$, which corresponds to 50 pulses from the pump laser operating at $1 \mathrm{MHz}$. Each of the individual pulse impinging on the particle will be scattered towards a large area MCP photomultiplier, positioned out 
of the propagation plane of the pump laser beam. In this near forward direction maximum signal for a variety of particle sizes can be expected.

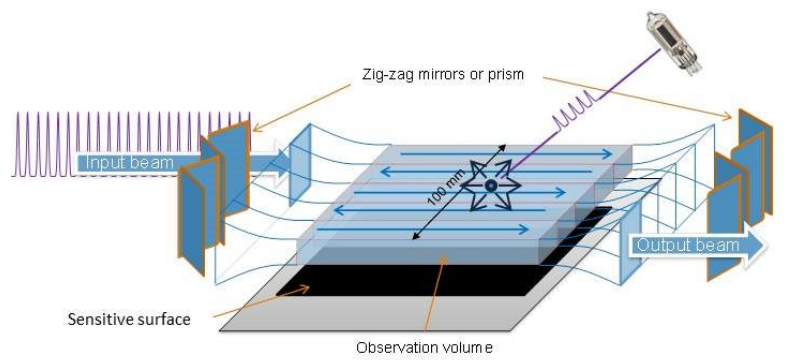

\begin{tabular}{|c|c|c|}
\hline \multicolumn{3}{|c|}{ LASER PARAMETERS: Fiber laser } \\
\hline Param & Value & Unit \\
\hline Wavelength & 355 & $\mathrm{~nm}$ \\
\hline Rep rate & 1 & $\mathrm{MHz}$ \\
\hline $\begin{array}{l}\text { Avg power } \\
\text { Energy pp }\end{array}$ & 30 & $\mathrm{~W} / \mathrm{mJ}$ \\
\hline Pulsewidth & 2 & ns \\
\hline \multicolumn{3}{|c|}{ DETECTOR: MCP } \\
\hline Param & Value & Unit \\
\hline Area & $>200$ & $\mathrm{~mm}^{2}$ \\
\hline Gain (G) & $1 \times 10^{5}$ & \\
\hline \multicolumn{3}{|c|}{ ELECTRONICS: ZI boxcar averager } \\
\hline Param & Value & Unit \\
\hline $\begin{array}{l}\text { Max input rep } \\
\text { rate }\end{array}$ & 450 & $\mathrm{MHz}$ \\
\hline $\begin{array}{l}\text { Min gate } \\
\text { width }\end{array}$ & 500 & ps \\
\hline
\end{tabular}

Figure 2 Description of the in-line particle detector block components

The signal acquired by the photomultiplier is further processed by means of the digital boxcar integrator, which performs a gated integration of the pulses and further averages only the signal, thus effectively increasing the signal to noise ratio of the detection.

The performance of the in-line detector can be evaluated by calculating the expected Signal to Noise ratio (SN), according to the relation $[11-12]$

$$
S N=\frac{G i_{c}}{\sqrt{\left(i_{\text {shot }}\right)^{2}+\left(i_{j s}\right)^{2}}} \approx \frac{G i_{c}}{\sqrt{\left(i_{\text {shot }}\right)^{2}}}
$$

where $i_{c}$ is the signal current at the cathode and $i_{\text {shot }}$ and $i_{j s,}$ are the current signal due to shot noise and Johnson noise, and being $\mathrm{G}$ the current gain of the photomultiplier. When substituting the expressions for shot noise and cathode current, Equation (2) becomes

$$
S N=\frac{G P_{s c a}(r, \lambda) \frac{e \eta}{h v}}{\sqrt{2 G^{2} e\left(i_{d}+i_{c}+i_{B G}\right) \frac{1}{\Delta t N_{p u l s e}}}}
$$

e being the electron charge, $\eta$ the detector quantum efficiency, h $v$ the photon energy, $i_{d}$ the detector dark current, $i_{B G}$ the signal current due to background and $\Delta t$ and $\mathrm{N}_{\text {pulse }}$ the pulse width and the number of pulses scattered due to the passage of a single particle. In Equation (3), the scattered optical power is calculated by Equation (1), while the number of pulses $\mathrm{N}_{\text {pulse }}$ is evaluated according to the relation

$$
N_{\text {pulse }}=D^{f_{R}} / v_{\text {part }}
$$


with $D$ the laser beam diameter, $f_{R}$ the laser repetition rate and $v_{\text {part }}$ the velocity of the particle. It is important to notice that according equation (4), for each particle crossing the observation volume, the detector will collect a train of equally spaced pulses, being the actual number of scattered pulses depending on the velocity of the particle.

By inserting Equation (1) and (4) into Equation (3) it is possible to evaluate the $\mathrm{SN}$ ratio for the detection process as a function of particle size and particle velocity. Figure 3 reports the results of the analysis conducted assuming the ideal case of negligible background contribution and nominal mirror reflectivity, and describes the detection sensitivity in a parameter space ranging from $20 \mathrm{~nm}$ to $100 \mathrm{~nm}$ for what concern the particle dimension, and from $10 \mathrm{~m} / \mathrm{s}$ to $100 \mathrm{~m} / \mathrm{s}$ for the particle velocity. The plots show that a $55 \mathrm{~nm}$ particle travelling at $100 \mathrm{~m} / \mathrm{s}$ can be detected by the instrument with a $\mathrm{SN}$ ratio of about 3 .
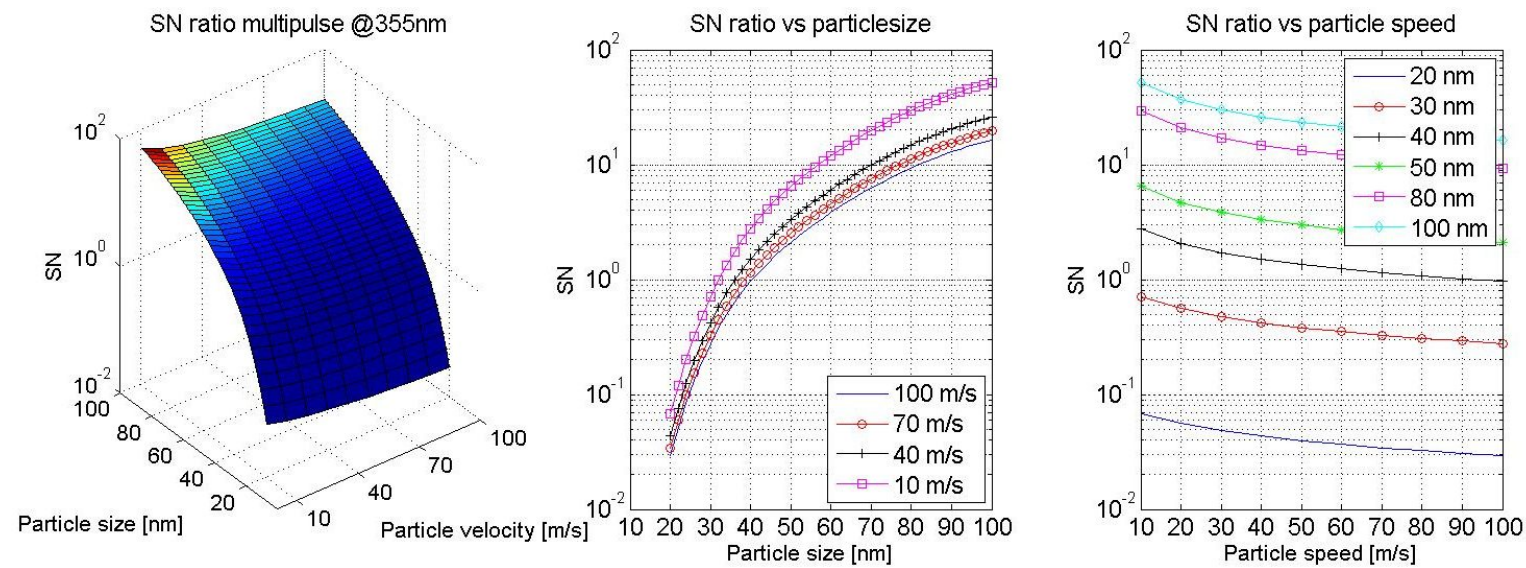

Figure 3 Calculated SN ratio for the in-line particle detector operating in ideal conditions.

Furthermore, Figure 3 also shows that the $\mathrm{SN}$ is strongly influenced by the particle size, while the dependency on the particle velocity is much weaker. Indeed, the SN is proportional to the inverse of the square root of the particle velocity, as apparent when substituting equation (4) into equation (3).

In the real case, the actual sensitivity of the instrument is degraded by the presence of background, which increases the noise at expenses of the signal to be detected. In addition, the reflectance of the mirrors used to achieve the coverage of the observation volume is expected to decrease due to aging and contamination effects [13], thus decreasing the power of the incoming beam and therefore the scattered signal.
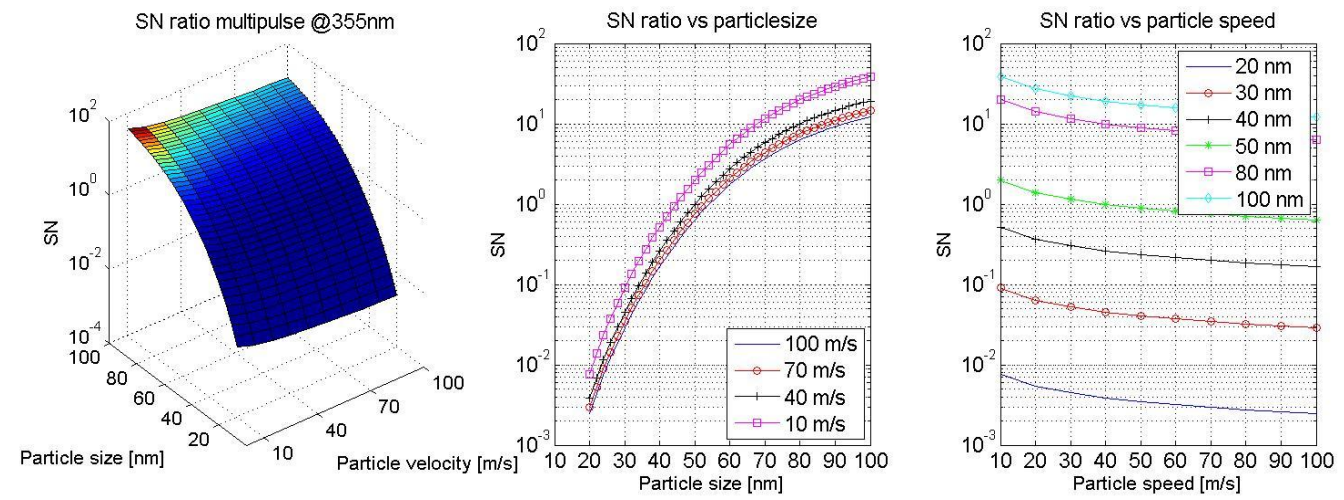

Figure 4 Calculated SN ratio for the in-line particle detector in presence of mirror degradation and background noise. For the analysis, a 1\% degradation per mirror and a background of $-130 \mathrm{~dB}$ are assumed. 
The impact of these two effects on the sensitivity of the in-line particle detector is apparent from the evaluation shown in Figure 4, which reports the performance analysis results calculated for the same parameter space of the ideal case but assuming a $1 \%$ reflectivity degradation per mirror and a background thirteen orders of magnitudes lower than the pump beam, mainly attributed to scattering from the mirror surfaces. Indeed, when these two effects are included in the model the minimum particle size detectable by the system assuming a threshold SN of 3 is equal to $65 \mathrm{~nm}$. These detrimental effects can be reduced by including in the design appropriate baffling and by ensuring the molecular and particle contamination on the mirrors are kept as low as possible.

\section{Capture rate and false positive estimation for the in-line particle detector}

The analysis conducted in the previous paragraph has provided the detector performance in terms of SN ratio. Unfortunately this parameter provides no information on the actual capability of the system of detecting all the contamination events with minimal false positive alarms.

In order to evaluate the expected probability of detection of a scattering event (i.e. the capture rate) and probability of false alarm, the distribution of the signals generated by the in-line particle detector needs to be taken into account [14].

In general terms, the expected output of the detector is an electrical signal composed by a train of pulses of variable amplitude, which are generated either by a scattering event or by noise. When a particle crosses the observation volume, a certain number $\mathrm{N}_{\text {pulse }}$ of pulses are deflected towards the photomultiplier according to Equation (4). A particle contamination event is considered detected by the instrument when $\mathrm{N}_{\text {pulse }}$ consecutive pulses of amplitude larger than a selected threshold are measured by the system. Pulses with amplitudes below the selected threshold are considered noise signal and therefore disregarded. The correct determination of the threshold is of paramount importance in order to achieve an efficient detection, which implies high capture rate and low false positive occurrence. These two parameters can be calculated, once the probability density of the signal $\left(\mathrm{P}_{\text {signal }}\right)$ and of the noise $\left(\mathrm{P}_{\text {noise }}\right)$ is known, by

$$
\begin{aligned}
& C R_{\text {mult }}=\left(\int_{V_{t}}^{\infty} P_{\text {signal }}(V) d V\right)^{N} \\
& F P_{\text {mult }}=\left(\int_{V_{t}}^{\infty} P_{\text {noise }}(V) d V\right)^{N}
\end{aligned}
$$

Equation (5) and equation (6) evaluate the probability that $\mathrm{N}$ consecutive pulse signals, respectively due to a scattering event and to noise, are larger than the threshold value $\mathrm{V}_{\mathrm{t}}$.

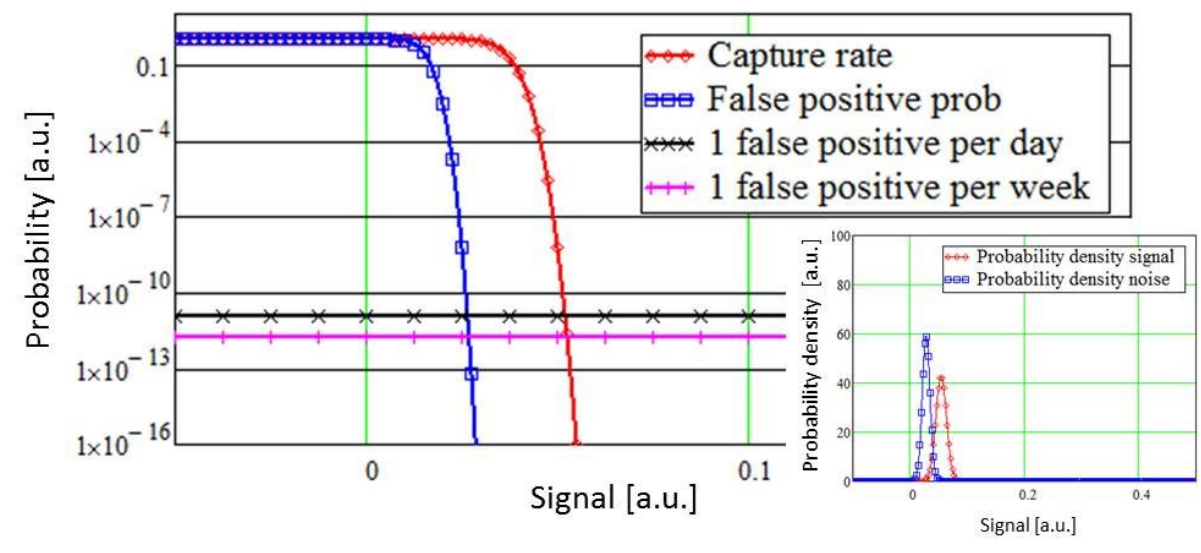

Figure 5 Capture rate and false positive probability as a function of signal threshold, assuming Gaussian distribution and a particle size of $66 \mathrm{~nm}$ 
The probability densities for signal $\mathrm{P}_{\text {signal }}$ and noise $\mathrm{P}_{\text {noise }}$ are assumed to follow a Gaussian distribution [12] where the average values are calculated on the basis of the signal generated respectively by a scattering event and by the cumulative signal obtained by summing background and dark current. In the same way, the standard deviation of the distribution is calculated considering the relative shot noise contributions.

An example plot of the expected capture rate and false positive probability is shown in Figure 5, for the settings including mirror degradation and background, and assuming a particle size of $66 \mathrm{~nm}$. Horizontal lines corresponding to two false positive requirements are also reported respectively in magenta and black with cross markers. The insert of Figure 5 shows as well the assumed Gaussian distribution $P_{\text {signal }}$ and $P_{\text {noise }}$ for a $66 \mathrm{~nm}$ particle size.

Figure 5 gives a graphical way of evaluating the expected capture rate of the system once the false positive probability is set. At first, the threshold signal value corresponding to the required false positive rate is calculated by the intercept of the requirement of false positive value (magenta and black lines in Figure 5 with cross markers) and the false positive probability function (blue curve in Figure 5 with open squares markers). This corresponds to solving equation (6) in the variable $\mathrm{V}_{\mathrm{t}}$ for the given false positive rate requirement. The capture rate is then readily obtained by the correspondent function curve evaluated at the previously derived threshold value (red curve with open diamond markers in Figure 5). It must be noted here that the adoption of a scheme based on the detection of multiple pulses for a single particle scattering event effectively helps in increasing the capture rate for a given false positive rate in comparison to the case of single pulse detection. This happens because it provides a mean of increasing dramatically the negative slope of the probability curves as a function of threshold signal.

The described procedure can be repeated for different particle sizes, in order to derive the expected capture rate as a function of particle size for a required false positive rate, which ultimately describes completely the performances of the in-line particle detector.

The results of this investigation are summarized in Figure 6, which report the capture rate as a function of particle size both in the ideal case (Figure 6a) and in presence of background and mirror degradation (Figure 6b) for three different particle velocities.
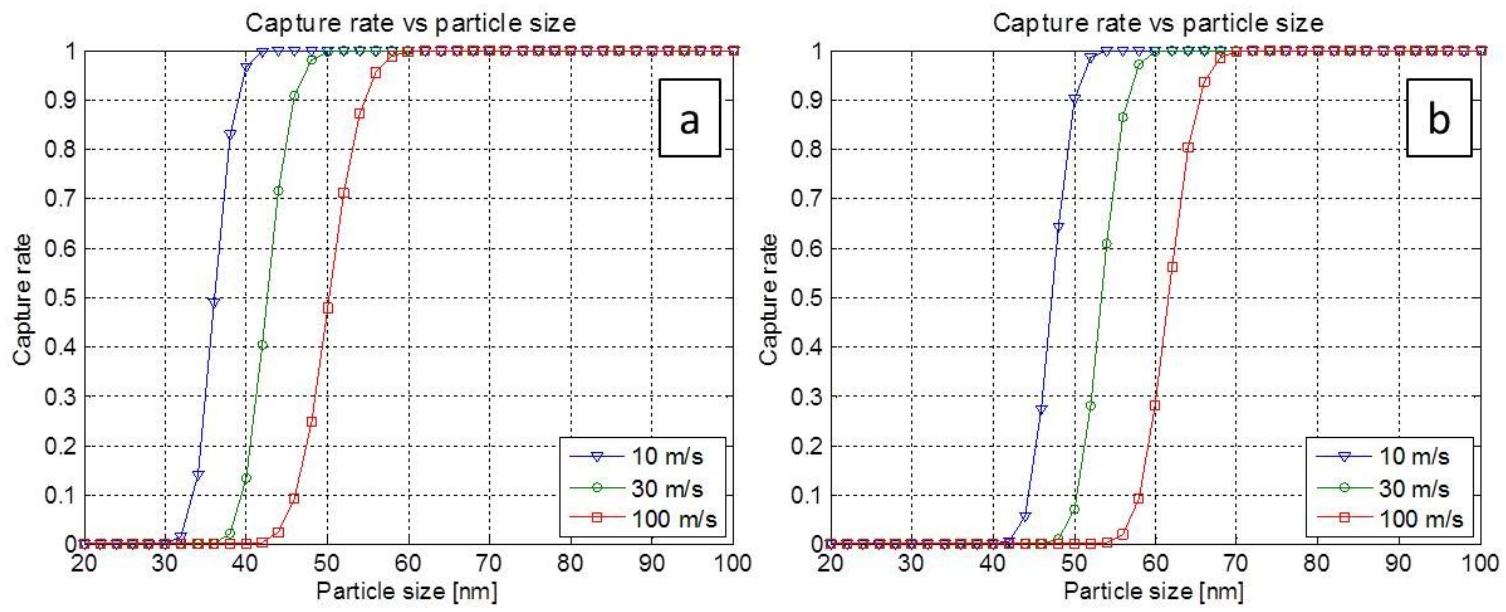

Figure 6 Detector capture rate as function of particle size for the in-line particle detector operating in ideal conditions (a), and in presence of background and mirror degradation (b)

The results reported in Figure 6 show that the in-line particle detector instrument operating in nominal conditions can sense $55 \mathrm{~nm}$ large particles flying at $100 \mathrm{~m} / \mathrm{s}$ and $40 \mathrm{~nm}$ particles flying at $10 \mathrm{~m} / \mathrm{s}$ with a capture rate of $90 \%$ and a false positive rate of one per week. In presence of mirror degradation and background, the sensitivity of the instrument is reduced, so that $65 \mathrm{~nm}$ particles at $100 \mathrm{~m} / \mathrm{s}$ and $50 \mathrm{~nm}$ particles at $10 \mathrm{~m} / \mathrm{s}$ can be detected with the same capture efficiency of $90 \%$. 


\section{CONCLUSIONS}

In this paper we have presented a design study of an instrument for the detection of fast flying nanoparticles based on light scattering. The instrument, based on commercially available equipment, has been specifically tailored for use in close environments where particle contamination on sensitive surfaces can degrade the quality of the process.

The design of the instrument is based on the use of high power high repetition rate laser operating at $355 \mathrm{~nm}$ as a pump source, and by a large area MCP photomultiplier to collect the pulses scattered by the particles and convert them in electrical signal. The proposed approach based on the use of high repetition rate lasers improves the discrimination between false positive and scattering events, because it is a lot more likely that a repetitive signal with $\mathrm{N}$ peaks is coming from a particle than from noise. As a consequence, the presented design allows high capture rates for the required false positive rate.

The performance analysis of the in-line detector has shown that the current configuration can efficiently sense particle as small as $55 \mathrm{~nm}$ with a capture rate of $90 \%$ and a false positive probability of one per week. Conversely, in presence of background illumination and mirror contamination, the instrument can detect $65 \mathrm{~nm}$ particles with the same capture rate

and false positive rate. Such decrease in performance of the instrument can be mitigated by proper design of a baffling system and by careful control of the molecular contamination on the optical components used in the setup.

\section{REFERENCES}

[1] Kohli, R. and Mittal, K. L., [Development of Surface Contamination and Cleaning: Particle deposition, Control and Cleaning], William Andrew, (2009).

[2] York, T. A., Evans, I. G., Pokusevski, Z. and Dyakovski, T., "Particle detection using an integrated capacitance sensor," Sensors and Actuators: A 92(1-3), 74-79 (2001).

[3] Feldman, M., [Nanolithography: The Art of Fabricating Nanoelectronic and Nanophotonic Devices and Systems], Woodhead publishing, 57 (2015)

[4] Fujimoto, J., Hori, T., Yanagida, T., Ohta, T.,Kawasuji, Y., Shiraishi, Y., Abe, T., Kodama, T., Nakarai, M., Yamazaki, T., Mizoguchi, H., "Development of laser produced plasma-based EUV light source technology for HVM EUV lithography,” Proc. SPIE 8322, 83220F (2012)

[5] Boheren, C. F. and Huffman D. R., [Absorption and scattering of radiation from small particles], John Wiley and Sons, (1998).

[6] Van Dijk, M. A., Tchebotareva, A. L, Orrit, M., "Absorption and scattering microscopy of single metal nanoparticles," Physical Chemistry Chemical Physics, 8, 3486-3495 (2006).

[7] Phillips, M. “Enabling EUVL for HVM Insertion”, International Symposium on EUV and Soft X-Ray Sources, 5 November 2013

[8] Koechner, W., [Solid state laser engineering], Springer series in optical sciences, (2006).

[9] Kume, H., Koyama, K., Nakatsugawa, K., Suzuki, S. and Fatlowitz, D., "Ultrafast microchannel plate photomultipliers," Applied Optics 27(6) 1170 (1988)

[10]Zurich Instruments, "UHF-BOX Boxcar Averager”, $2014<$ http://www.zhinst.com/products/uhfli/uhf-box>

[11] Yariv, A., [Optical electronics in Modern Communcation], Oxford University Press, (1996)

[12] Lesurf, J. C. G., [Information and measurement], IoP publishing, (2001)

[13] Tighe, A., Pettazzi, F., Alves., J., Wernham, D., Riede, W., Schroeder, H., Allenspacher, P., Kheyrandish, H., "Growth mechanisms for laser induced contamination on space optics in vacuum," Proc. SPIE 7132, 7132L (2008)

[14] Barrett, H. H., Swindell, W.,[Radiological Imaging: the Theory of Image Formation, Detection and Processing], Academic Press, 1981 